\title{
MAKING INTERACTIVE TV EASIER TO USE: \\ INTERFACE DESIGN FOR A SECOND SCREEN APPROACH
}

\author{
LEON CRUICKSHANK, EMMANUEL TSEKLEVES and ROGER WHITHAM \\ Brunel University, Uxbridge, UK \\ ANNETTE HILL and KAORUKO KONDO \\ Westminster University, Harrow, UK
}

KEYWORDS: interactive TV, PDA, user interface, second screen

nteractive television (iTV) has the potential to revolutionize the way we consume broadcast media,
but users still find both the notion of iTV and the services currently available problematic. This paper describes a project that investigates a representative group of users' aspirations, and barriers to iTV service engagement in the UK. This primary research informed the development of new User Interface (UI) and service solutions that addressed these barriers. Specifically, a second screen solution was developed to remove the need for iTV services to use on-screen graphics, dramatically improving the possibilities for effective interaction and navigation for iTV interfaces and services. The effectiveness of these solutions was evaluated through the testing of these new iTV services in a representative group of family homes.

\section{INTRODUCTION}

By 2012 all TV broadcast in the UK will be in digital format with a region by region switch-off of analogue broadcast starting in 2008 (Department of Culture Media and Sport, 2007 [website]). US conversion is set for 2009 and the Geneva 2006 Agreement (International Telecommunication Union, 2006 [website]) means that in 2015 legal protection of analogue TV transmission along a country's borders will be removed in Europe, Africa and parts of Asia with a complete transition to digital broadcast by 2020 .

The move to digital from analogue offers some remarkable possibilities for viewers, broadcasters and programme makers. In addition to improvements such as choice (the UK currently has only five analogue broadcast channels) and an improvement in picture quality and sound, there is a range of possibilities for new types of content. These include interactive services, data casting and niche/community broadcast channels. In essence, 
anything that can be represented in digital form can be broadcast, ranging from computer programs and websites to instructions for home automation systems.

Research within households tells us the practical development of these possibilities is being stifled by a lack of engagement with current iTV services by users. The overarching aim of the research on which this paper is drawing is the identification and resolution of these problems with user engagement in iTV. We argue that the control and access to interactive content is restricted by current interaction models, that is, the conventional remote control (see England \& Finney, 2002; Eronen \& Vuorimaa, 2000; and Steemens, 1998). This was supported by our experience in the fieldwork but clearly this is a complex picture informed by the services on offer and their marketing, user perceptions and willingness to engage with new technology. A more sophisticated form of input and control needs to be introduced for iTV to reach its full potential.

We present research that tested the use of personal touch screen devices, in this case Personal Digital Assistants (PDAs), as a mechanism for accessing iTV services and controlling television operating systems (for example, Electronic Programme Guides (EPGs)) without having to interrupt broadcast programmes at all. The objectives of this research were:

- To identify some of the barriers to usage of current digital TV systems through a series of interviews in the homes of a representative sample of the current users of digital TV;

- To design technically realistic, near-future iTV services and infrastructure led by this initial investigation; and

- To test our hypothesis that new modes of interaction can help to remove barriers to the use of iTV services by taking simulations using live TV into the homes of study participants.

\section{RESEARCH METHODS}

Throughout this project qualitative methods were mainly employed, using interview and participant observations. We selected 20 households (62 people) in different socio-economic groups - ABC1 C2DE (Bocock, 1993) from the Greater London area. In addition to socio-economic status this sample represented a cross section of household size, from single occupants to families of 6.17 out of the 20 households subscribed to Sky (a UK satellite service) and 3 households subscribed to Telewest (a UK cable TV service).

Households were visited three times. The first two visits were made to build a trusting rapport with the participants and to study the overall media consumption within each household. The initial visits normally lasted between 60 and 90 minutes, depending on the number of people in a household. The second visit was carried out a month after the first. Here participants were not only interviewed but were also asked to demonstrate how they actually use TV remote controls, EPG services, interactive TV content (games, shopping, information, etc.) and the Internet.

The third visit involved the installation and testing of new prototype iTV interfaces and hardware systems developed in response to the initial visits. 


\section{RESULTS: INTERVIEW}

One of the most important aspects of the project was to understand how users perceived the new medium of interactive TV. For our participants interactivity meant: 'interacting with friends', 'more options', 'select and control', 'saving time', 'more information', 'personal service', 'giving you choices'. This is on the understanding that it can be applied to almost anything users do whilst they access programmes or websites.

The exploratory study also revealed that interactivity is only perceived as a positive concept when it is safe and secure. As Adel says, 'interactivity can be a good thing, but only if it is safe', indicating that there are risks to using new technology. This perception was also echoed by several other respondents. For instance, Rickey states that, 'I don't think I'll use it for playing games or gambling', when speaking of his concern about the security issues involved with the new technology. According to Sally, a 30-year-old sub-editor, 'It means you are places, things like voting, buying and gaming. Some [are] more complicated. I like dramas, I use [interactive services] to get more information and stuff'. For her interactivity takes the viewer to 'places' away from the main space of the TV programme or channel, to a space designed for consumers to buy things or to gamble.

Most of the respondents used few functions available from their iTV services, preferring to focus on familiar features suitable for their needs, rather than trying out a wide range functions. The EPG was regarded as the most useful interactive function as it allows people to navigate through the multi-channel environment. As a study participant put it, 'You just look at what you've got now, and find what is on now.'

The 'reminder' function was also popular amongst respondents as it was thought to be easy to use. This function (provided by both Sky and Telewest) allows users to flag programs they wish to watch from the EPG using the remote. According to Mark, an 18year-old student: "I like that a lot, I can never be bothered with the $[E P G]$... I just set the reminder, and then it comes on three minutes before the programme starts'. Similarly another respondent found the reminder function very convenient. She said 'if we are watching something else, and I put it on, and it comes up and kids say, "Mum, it will be in 5 minutes", However, other respondents did not know how to control the reminder function. For example 'I can't get the reminder, and can't get rid of it, it actually stays there. So I am funny about using it, because if I use it, I can't watch other programmes'.

The issue of design was dominated by participants' attitudes to and experiences of interactive media: participants' positive or negative experiences of using interactive functions framed their responses to the design of iTV functions and services. In terms of interactive television, participants most wanted the functions to be designed to offer the quickest route to content, and not take the user away from the main viewing experience. For example, the reason that participants liked the EPG was because it is simple to use and offers a direct route to TV programmes. Conversely, the reasons respondents gave to justify their dislike for the $A-Z$ listings in the EPG were centred on it being confusing, taking too long and the picture often freezing. 


\section{RESPONSE TO THE FIRST TWO VISITS}

Our initial investigation provided a range of insights into the way users of iTV think about and interact with TV and interactive services. These insights led us to consider how we might investigate further these issues in a third and final visit to study participants' homes.

\section{SECOND SCREEN APPROACH}

A key theme identified in the first study was the conflict between the core functions of TV systems, namely displaying the TV picture itself, and the auxiliary functions which support them. Many of our study participants valued greatly the information services offered by their iTV systems, such as the EPG, but the need for these to occupy the same display space as the main image was a cause for conflict.

This led us towards the concept of introducing a portable second screen to the iTV interaction mechanism. A portable second screen offers the opportunity to remove the need to show UI elements on the main television screen. This avoids many of the problems related to UI latency/reaction speed in iTV systems; viewers are not going to miss broadcast information due to an interactive service not responding rapidly enough. Users told us slow UI responses are especially frustrating when exiting from iTV services back to the normal picture, a problem that a second screen obviates immediately.

A second screen approach also offers the opportunity to extend the model of iTV operation by offering a far richer interaction mechanism than conventional remote control devices. It also allows for the dynamic reconfiguration of the UI to reflect a user's needs and desires. This could be a matter of personal preference or could represent a major enhancement of possibilities for those with significant impairments which affect their interaction with iTV services.

\section{The control device}

With a move away from infrared communication between $\mathrm{TV} /$ set-top box and remote, the control device would not have to be pointed directly at the television set to function, allowing it to be used in a more casual 'lean back' position or even from a different room. This is another facet to iTV where broadcast programmes and iTV services co-exist in a parallel relationship rather than one having to displace the other on a single television display.

While the role of the TV remote control has changed significantly over the past decade with the advent of more complex TV content, the domestic television remote control has changed little. There are many commercially available remote controls which break the traditional mould, such as Philips Pronto, Pacific Neotek's OmniRemote, the OneForAll URC-9990 Mosaic, Marantz's RC9500 and Logitech's Harmony 1000. Such devices allow for the mapping of multiple operations to one soft keystroke, however they do not interact with iTV services in a different mode to traditional television remote controls.

Crucially these 'universal' remote control devices do not have the ability to interface directly with broadcast content, including the custom interfaces used in iTV services. As such these devices are an aggregation of conventional remote functions rather than offering new iTV possibilities. 
A study closely related to ours was on an integrated use of a PDA, TV and set-top box to demonstrate an estate agency service (Robertson et al, 1996).

Although this research was concerned with detailing guidelines for iTV interaction via a portable touchscreen device it did not focus upon interaction with the TV as a whole, instead concentrating on only a single iTV service. There have also been projects associated with the use of touch pads (but not touchsensitive screens) as part of remote controls (Enns \& Mackenzie, 1998) and on using PDAs to control household electronics with both stylus and speech input (De Vet \& Buil, 1999; Nichols et al, 2002), plus projects that control TV in a multi-user context with mobile phones (Park et al, 2006). Interestingly, none of these systems, prototypical or commercial, seek to employ portable second screen devices to change the core iTV user interaction model for exploring available content and consuming iTV services in a cohesive and user-friendly manner.

\section{Touch-screen interaction}

We investigated a touch-screen mode of interaction as likely to support a suitable degree of deviceindependence for our research outcomes. It has already been established that portable touch-screen devices such as PDAs can work closely with iTV services to take advantage of the benefits of both devices, the TV offering high quality visual/audio output and the PDA offering interaction through complex input and the display of simple graphics, such as floor plans in the estate agent service (Robertson et al, 1996).

There is also evidence that using a PDA for interactive activities around the home can result in a 50 per cent reduction in time spent, with a 50 per cent reduction in error rate compared to using the device's standard (though not necessarily remote controlled) UI (Nichols et al, 2002; Nichols, 2001). Such dramatic benefits are not necessarily to be expected for television as they have high quality displays, which can provide good user feedback, unlike the primitive monochromatic displays of some household appliances. Looking at the use of PDAs in interactive situations in the home De Vet \& Buil present research that describes the development of a UI that does not simply replicate the conventional hardware remote layout using a PDA screen (De Vet \& Buil, 1999). They note the key advantage we have expanded upon: the possibility of remote device control, specifically with respect to television, without the need to disturb the viewing of others.

This is not to say that small touch-screen devices such as PDAs are without UI drawbacks; the small screen, lack of a full-sized keyboard and the lack of tactile feedback from the screen are all issues which must be addressed. Much research looks into the challenges of information visualization and interaction under such constraints, too much to cover in detail here. Relevant areas of concern include the development of semi-transparent widget overlays for visualization of information (Kamba et al, 1996) and employing various strategies to mitigate the problems of small screen web browsing (Wobbrock et al, 2002). Such strategies include variable zoom on different areas of a document or UI, Rapid Serial Visual Presentation (RSVP) of text and the exploitation of hardware buttons.

\section{EPG interaction}

Our initial investigation also informed our decision that the EPG would be the focal point of our 
prototype system. We found that much of the research in this area is based around traditional hardware remote controls with a number of studies noting the need for improved usability in EPGs and iTV products (Black et al, 1994). Daly-Jones and Carey note specifically the problems with hardware remote controls, including poor mapping of functions between remote and display, poor feedback for users actions with the remote and invisible functions found only on the remote and not represented on screen (Daly-Jones \& Carey, 2002). There have been some novel solutions for problems associated with EPGs including (Eronen \& Vuorimaa, 2000) which tests new EPG models based around an infrared remote and TV. Two other studies (Smyth and Cotter, 2000; Isobe et al, 2005) investigate automatic personalized recommendation systems for TV programmes to filter unwanted content.

\section{PROTOTYPE DEVELOPMENT}

Responding to the two sets of home visits and the existing research as described above, we designed a system that addresses some of the identified barriers to user engagement with iTV services. This system consists of physical or hardware components, new user interfaces and (outside the scope of this paper) supporting software infrastructure.

\section{User interface}

In this design we were led by our initial research broadly in terms of the removal of text from the main viewing screen, but also in details such as the use of channel logos for channel identification. We also exploited the high resolution of the screen in the device selected (200 ppi) to give us more flexibility in the graphic design of the UI.

The interface operates in landscape orientation; this allows a horizontal time axis on the EPG and therefore more items per channel to be shown. The default view on start-up (shown in Figures 1 a and 1b) can be broadly split into three horizontal areas of activity.

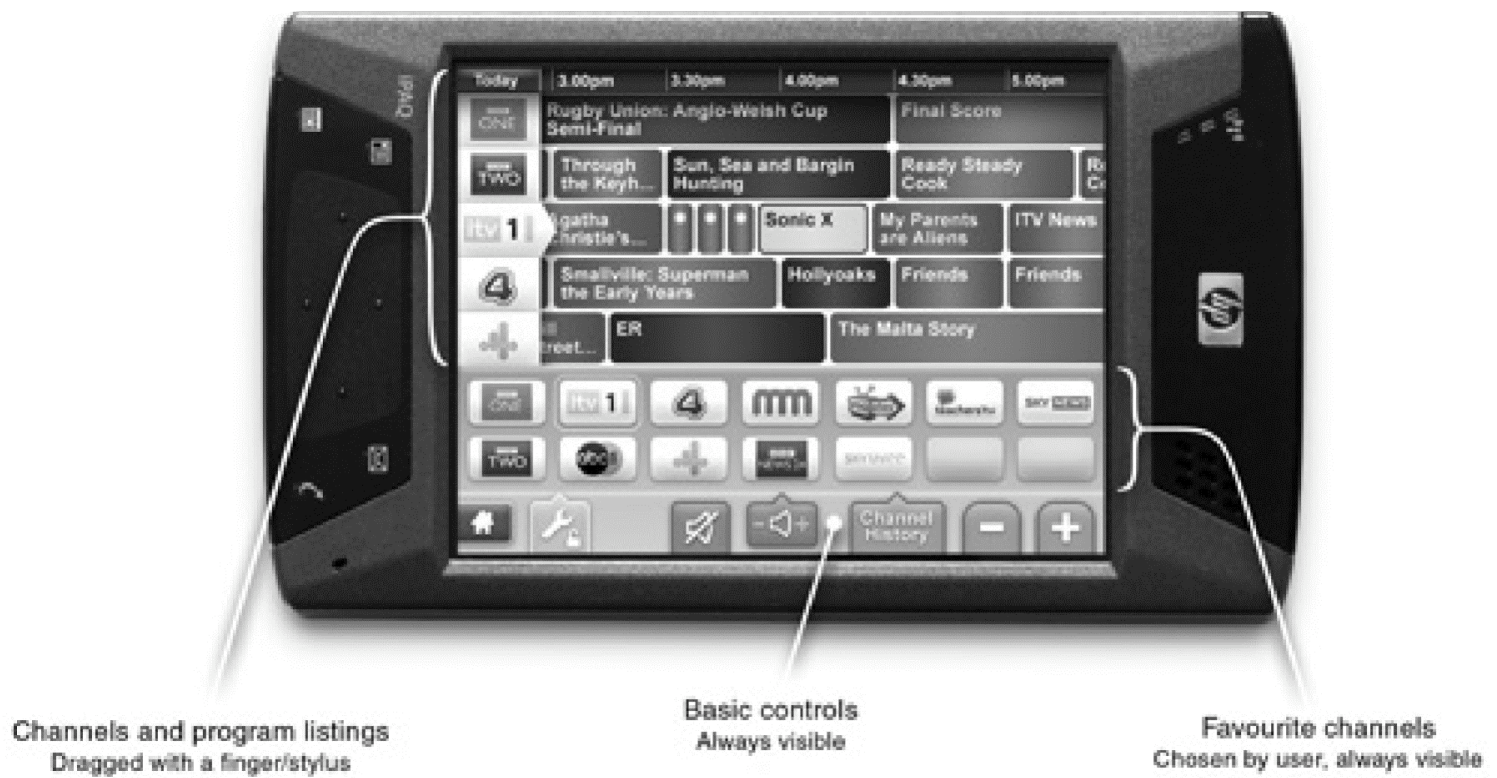

Figure 1a. Initial state of the PDA UI. 


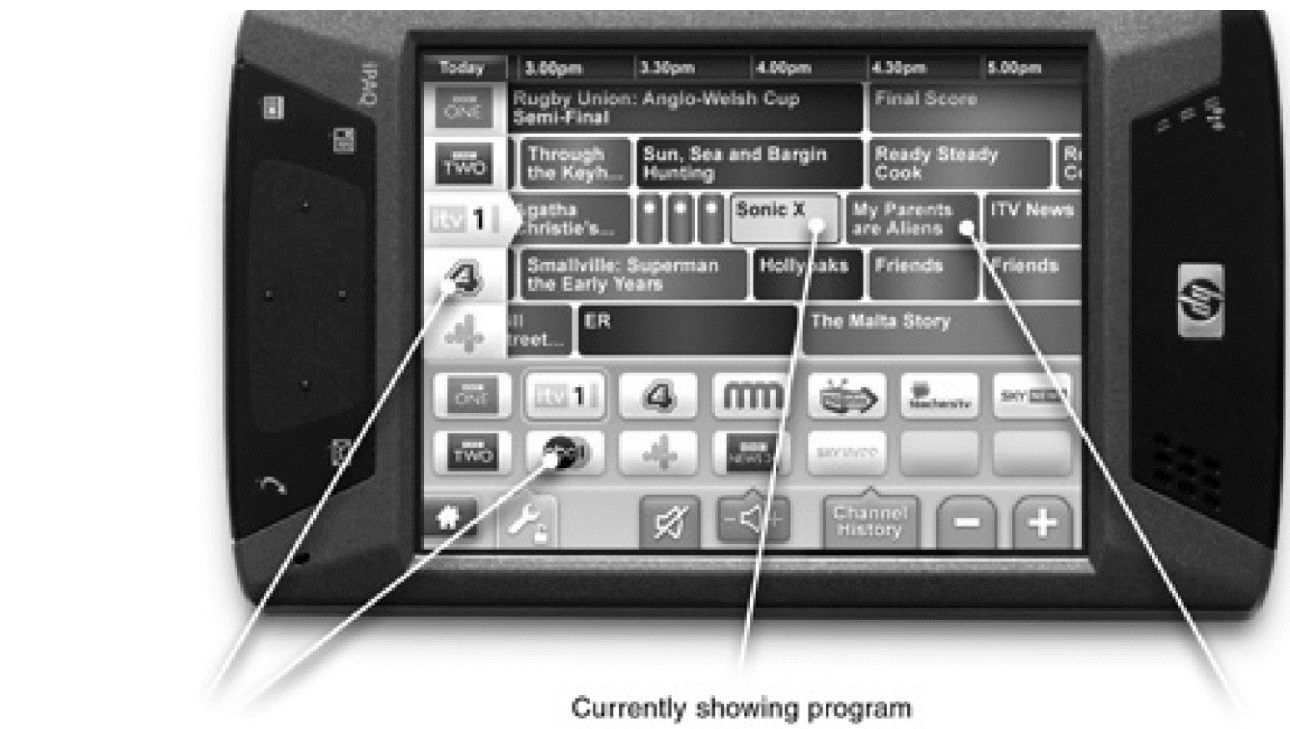

Tap logos to change channel

Program to be recorded

Figure 1b. Further details of the PDA UI

At the bottom of the layout there is a global bar; this is always visible during any operation and provides instant access to the basic remote control functions (volume, mute, channel +/-) along with a channel history. The latter was included due to comments from our initial study in which users expressed a desire to be able to navigate back to previous channels without having to go back into the EPG, remember what channel they were watching and scroll to that channel. The channel history keeps a rolling record of the last five channels visited, much like a web browser (see Figure 2).

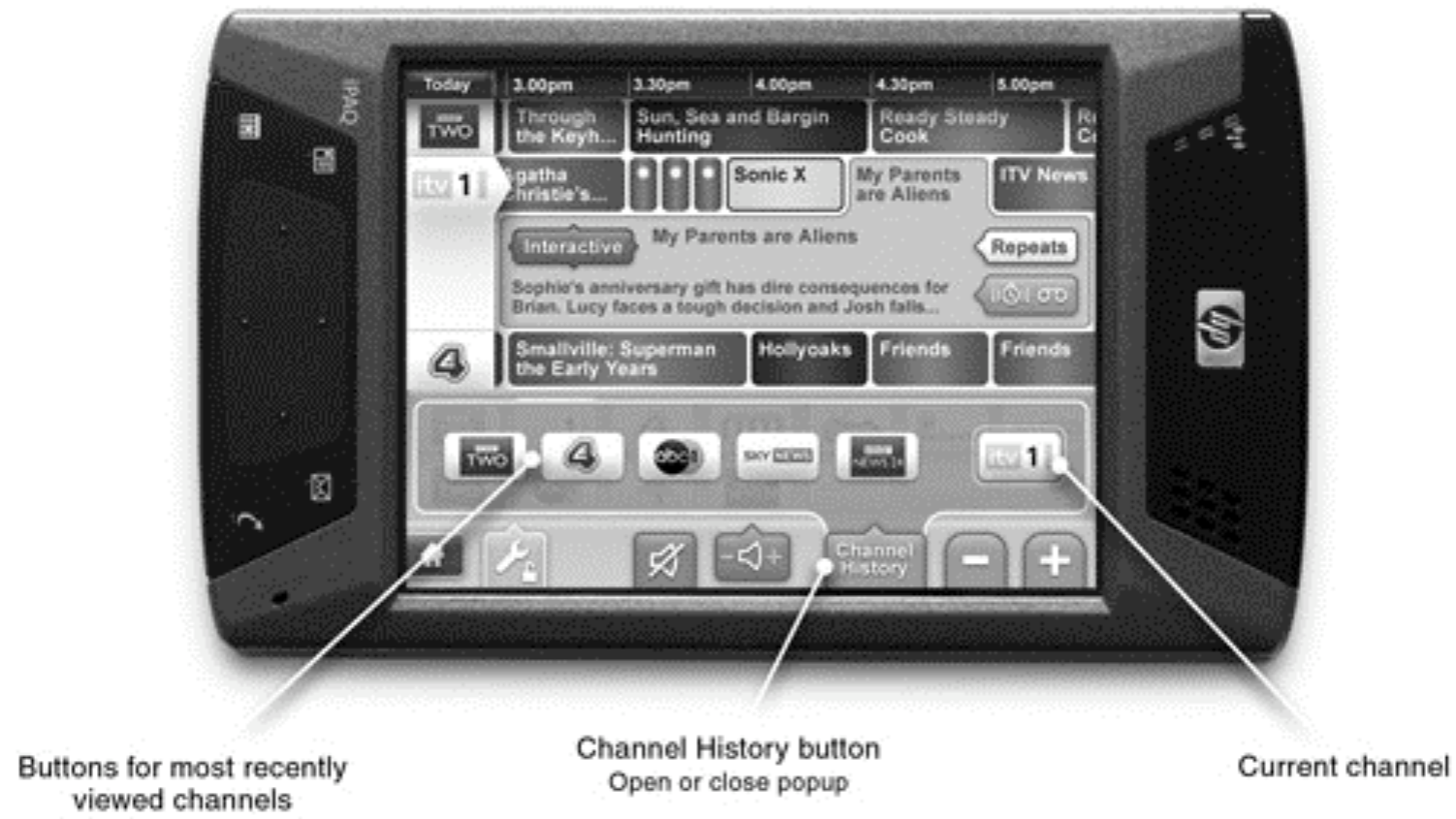

Figure 2. Channel history expanding menu. 


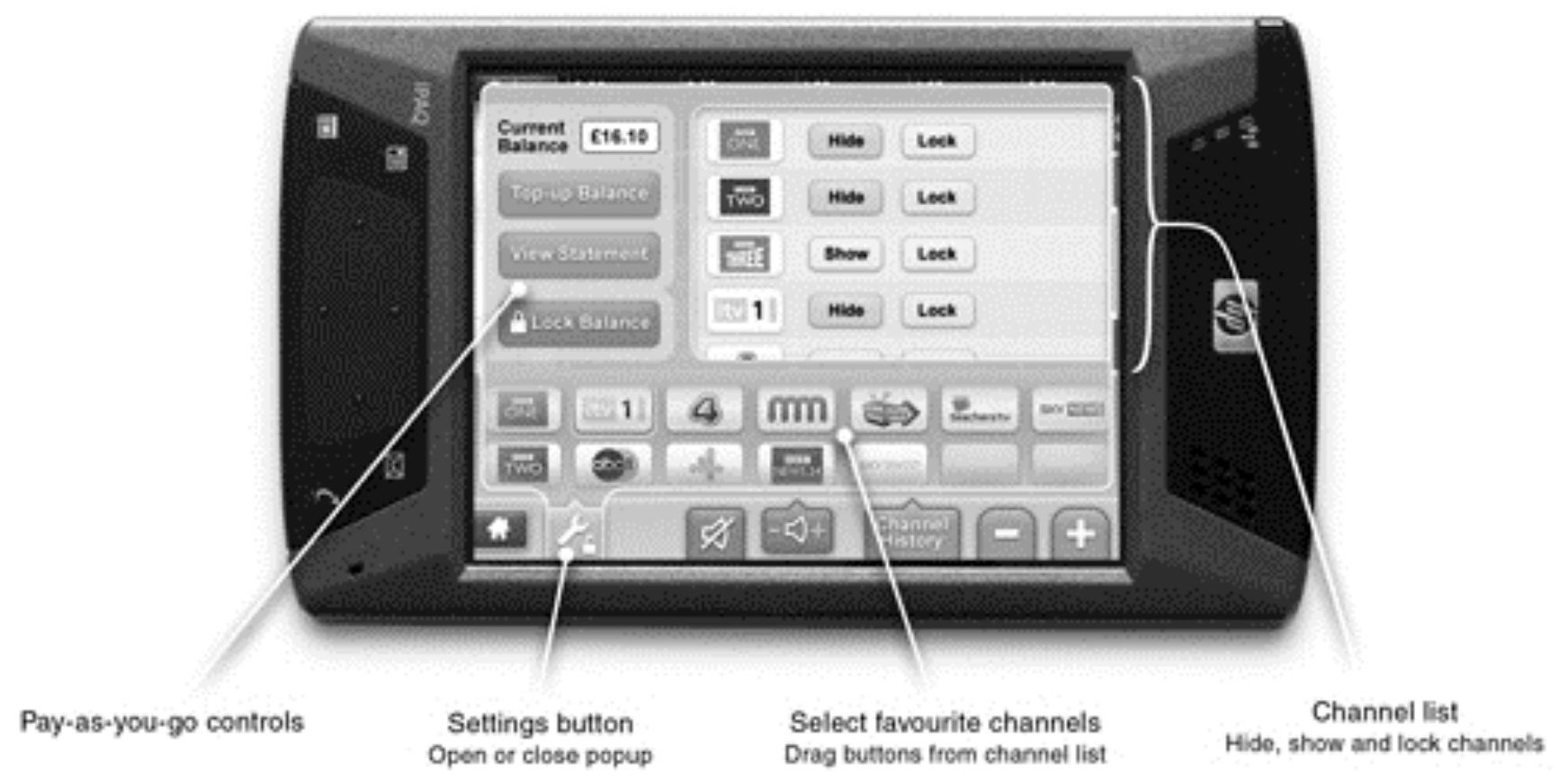

Figure 3. User settings and log-in.

Above the global bar is the 'favourites' area containing a number of buttons for one-press access to channels, identified via channel logos. Users' favourites can also be dynamically added/removed in the settings section (see Figure 3). A full implementation would be likely to include a simple user-switching feature to allow several groups of user-specific favourites to be maintained.

The EPG occupies the upper half of the layout; this has a horizontal time axis with icons to represent channels. Pressing these icons changes the channel on the television, just like the buttons in the 'favourites' area. Tapping on a particular programme in the EPG expands the item to show additional information in the EPG including a programme description, record/remind functions and access to interactive services if available (see Figure 4). A second tap within the expanded programme returns it to its original state.

The EPG uses a drag-and-release interaction to navigate around the plane of programmes. This increases the real estate available for content display as it removes the need for scroll bars, while also reducing visual clutter. Tapping the home button (left-hand end of the global bar) takes the EPG to the current time and programme being viewed on the television.

Dragging the EPG is innovative but potentially problematic in that this control mechanism is not made visible in the UI. 


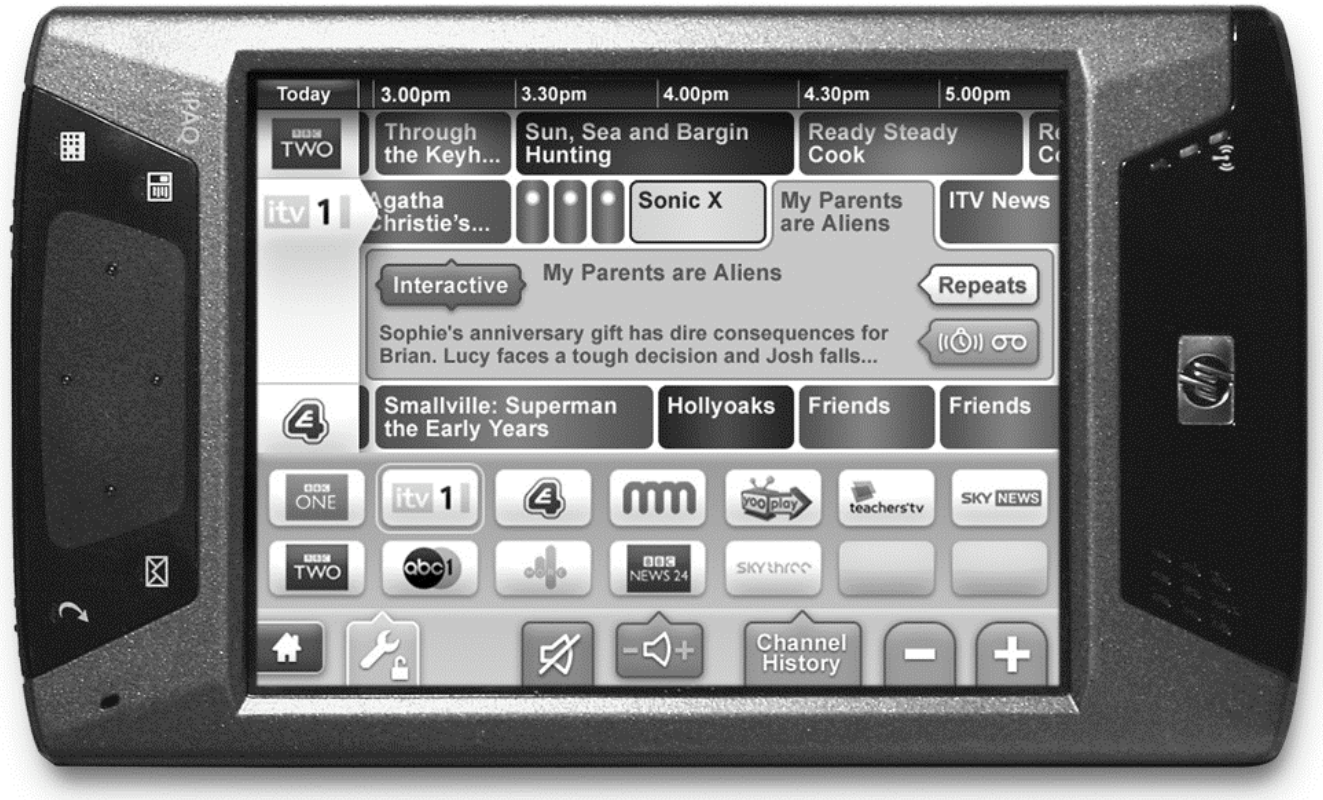

Figure 4. Additional programme information.

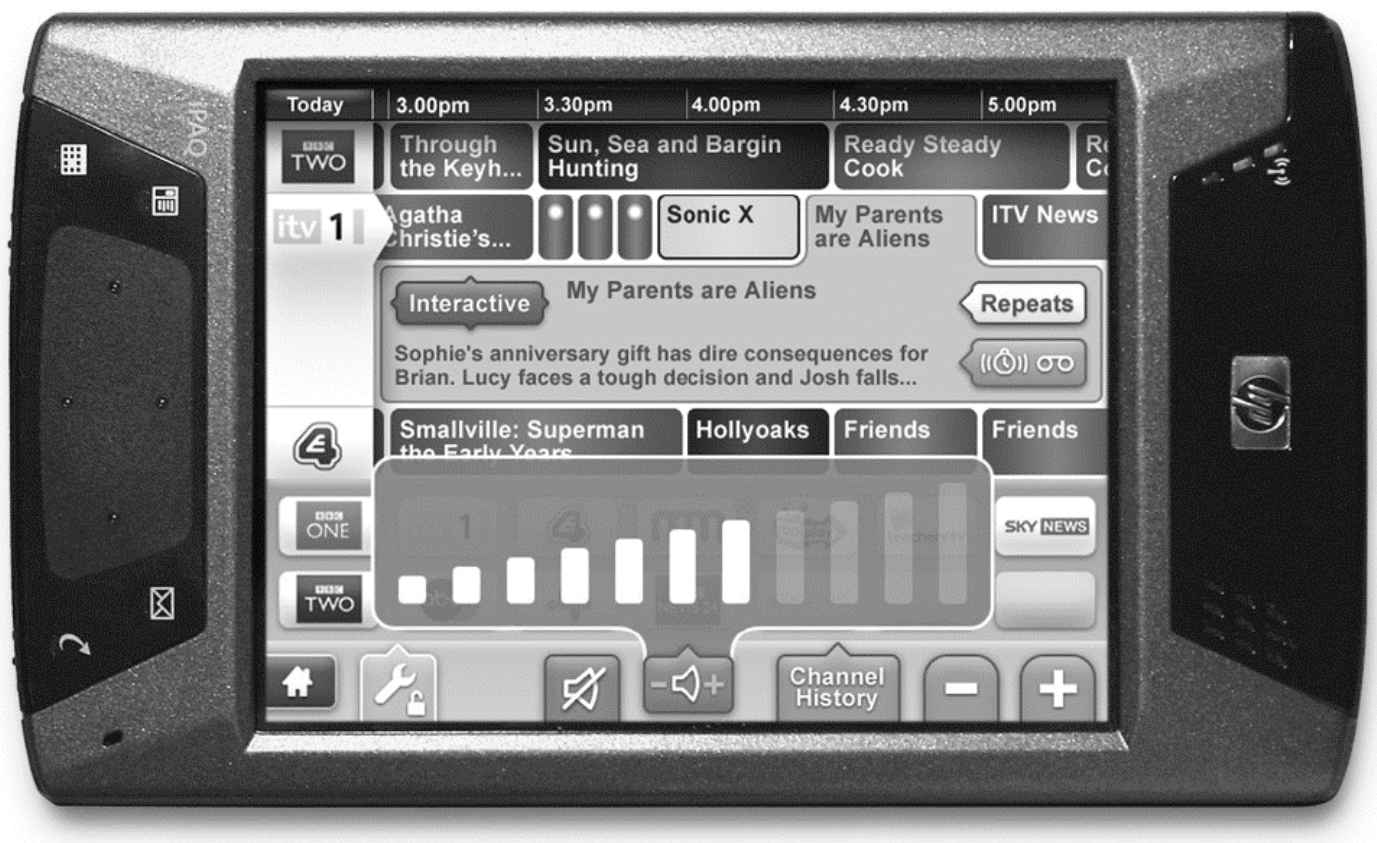

Figure 5. Expanding touch-sensitive volume menu.

Additionally, during dragging there is inevitably a high degree of screen occlusion by the hand/finger if a stylus is not used. The first problem is mitigated by the ease with which the interaction method can be learned. The second problem applies not only to dragging the EPG, but also to the entire UI if it is to be finger-operable. It can be solved to some extent by creating buttons that provide visual feedback that can be seen around the sides of the finger as they are pressed. A special realization of this principle was developed for programme-specific functions (remind, record, repeats), channel history, the settings menu 
and volume (see Figure 5). These 'popup' menus are semi-transparent to help reinforce their transient nature while providing access to additional functions in a space-efficient manner (Kamba et al, 1996).

\section{HARDWARE}

Our prototype consisted of a laptop computer capable of receiving Freeview TV that could be connected directly to participants' TVs. The laptop could function in two distinct modes: the first allowed the TV channel to be changed using the PDA connected to the laptop via Bluetooth; the second allowed control over the laptop via a generic infrared iTV remote control. This set-up allowed us to perform evaluations using either the PDA or a traditional onscreen UI using the same basic hardware.

Both the on-screen UI and the PDA UI used standard TV-Anytime metadata (Bleb.org, TV Anytime, 2007 [website]). This allowed us to provide a real time EPG as part of the PDA and on-screen UI. This, combined with live Freeview TV, made our prototype simulations as transparent as possible.

\section{TESTING METHODOLOGY}

To establish the validity of our analysis of the initial interviews we returned to the sample of households from our first two visits and undertook a series of structured and unstructured interviews/focus group sessions, each with audio and video recording of the users' interaction with the iTV services via our second screen and on-screen prototypes.

Participants were asked to interact with two sets of iTV service simulations, an EPG and a music video clip voting application (these voting services are not documented in this paper). Users were asked to interact with the same services, operating them using both of our two prototype modes: with a traditional remote control and with our PDA remote control.

Users were instructed in the use of both prototype modes for a few minutes at the start of sessions. We initially supplied and demonstrated the PDA without a stylus; during the session we asked users whether they would like to use a stylus and supplied one if requested.

It should be noted that there were some variables that interfered with the effectiveness of the testing. Freeview reception, surprisingly, is not universally available in all areas of Greater London, which required the reversion to a backup of displaying a still image of the chosen channel in a few cases. This compromised the quality of the simulation as a believable iTV service in those houses not controlling live TV with our system. Also due to hardware performance problems, our prototype's live television was limited to approximately 15 channels. Performance problems exclusive to the PDA meant that the EPG portion of the UI had to be visually simplified, resulting in the removal of rounded corners and background gradients from programme items in the EPG.

\section{RESULTS AND DISCUSSION}

Initial results indicate that user reaction towards the presentation of the EPG on a second screen is very positive. The majority of the participants acknowledged the usefulness of employing such a handheld device to display and interact with the TV guide while leaving the main TV picture free of any graphics. Teenagers and women were the most enthusiastic users of the system finding it more user- 
friendly and straightforward when compared to the conventional on-screen iTV UI. Example responses include Annette's: 'I think it makes more clear what is going on...I think it's quite easy to use'; and Margaret's: 'I prefer this to the one on the screen, I'd prefer to have this.'

There was doubtless a novelty factor at play when participants evaluated the PDA against the more traditional on-screen UI. This was especially evident in the reactions of children and teenagers, for instance Janette's son (14 years old) said, 'Oh, this is great... I'll probably get one of these for Christmas.'

It is very interesting that, although a portable gadget such as the PDA would seem likely to appeal more to male respondents (as one of the study participants pointed out, 'I think this gadget would be more popular with the fellows'), in fact, it was women who especially found the PDA system and mode of interaction preferable and were very keen to adopt it. For example, Diane says, 'I don't know why but this feels less threatening. I find it easier.'

Men on the other hand seem to be more sceptical about the introduction of such handheld devices in their living room environment and were especially concerned about its pricing. Paul says 'I think it's expensive for people to buy that', while Mark comments "If you lose the remote control [it would cost you $£ 10$ ], if you lose this it'll cost you a lot.'

Another concern raised by several male respondents was in response to direct interaction with the PDA. When a stylus was present it was generally easier for them to interact with the device, compared with when they initially attempted to control the PDA with their fingers, as illustrated by Angelo: 'I would say you [would] probably be better off with a stylus, especially for someone like me with dummy fingers'; and Peter: 'I think it responds better with a pen, with a finger it did not respond quite so well'.

Participants also commented on the superior legibility of text on the PDA screen, as explained by Diane: 'Oh [the text size is] fine, because it's here and not all around over there then that's fine. I can read that no problem'; Mike: 'I like this its great, in a way it's better [than the TV screen] cos you've got it in front of you like a book'; and Margaret: 'I can read [the PDA] better... I need new glasses but I can read this anyway'.

While this preference for the second screen device test could be attributed to the much higher physical resolution of the device we suggest that the mobility of the PDA also played a part. Moving the device nearer and further from the eyes was a common observation in our study, suggesting users could easily suit their own reading distance preferences.

A note of caution needs to be introduced here. We developed a parallel set of interfaces, one for conventional remotes and one for second screen to mitigate the novelty factor in terms of interface, and the feedback we received identified specific factors that were an improvement on conventional viewing. The degree that novelty played in the positive responses we received for this system is an open question without a long-term study that was outside the scope of this pilot project.

\section{CONCLUSION}

We believe we have demonstrated that from a user's 
perspective that there are significant problems with the current design of UIs and services for UK iTV products. A new approach is required that goes beyond the hard button remote control paradigm if iTV services are to be truly successful. The second screen system we have developed and tested offers significant advantages over conventional remote controls when using iTV services: users show a strong preference for this system over conventional remote controls, something we attribute primarily to the mobility of the PDA and its superior visualization of service information.

Reservations about the PDA system were recorded, linked both to the perceived cost of the units and the difficulty of finger-based interaction with the touch screen. As the technology develops, costs will decrease. It is likely that a commercial realization of this approach would utilize the user's mobile phone as a display and control device. In the UK, Sky have a very rudimentary control by phone system, however it was in only the last few months of the study that mobile phone technology had sufficient processing power to meet the technical needs of our prototype hardware.

By using an 'intelligent' second screen for displaying interactive elements and for TV control we provide an uninterrupted broadcast experience on the TV itself, addressing an important concern highlighted in our first study, while providing superior interaction with iTV services for iTV users.

The flexibility of a second screen allows for the potential for individual and modifiable interfaces to control universal broadcast iTV services. While this may be interesting for the everyday individual it has profound implications for those with disabilities and those providers who in the UK under the Disability Discrimination Act (1995) are legally bound to provide an equivalent service to all users. We are currently working with the BBC and others in developing a longer-term project that provides for longer term in-home testing of technologies for increasing the accessibility of iTV.

\section{REFERENCES}

Black, A., Bayley, O., Burns, C., Kuuluvainen, I. \& Stoddard, J. (1994). 'Keeping viewers in the picture: real world usability procedures in the development of a television control interface'. In Plaisant, C. (Ed.) (1994). Conference on Human Factors in Computing Systems: Conference Companion CHI 1994. Boston, Massachusetts, USA, 24-28 April 1994, pp.243-244. New York: ACM Press.

Bleb.org (2007). Bleb.org [internet]. Available at: http://www.bleb.org/tv/ [accessed May 2007].

Bocock, R. (1993). Consumption (Key Ideas). London:

Routledge.

Daly-Jones, O. \& Carey, R. (2002). 'Navigating your TV: The Usability of Electronic Programme Guides'. UsableiTV, 3, pp.26.

De Vet, J. \& Buil, V. (1999). 'A personal digital assistant as an advanced remote control for audio/video equipment'. In Brewster, S.A. \& Dunlop M. D. (Eds.), Proceedings of the Second Workshop on Human Computer Interaction with Mobile Devices. Edinburgh, Scotland, 31 August 1999.

Department of Culture Media and Sport (2007). Digital

Television [internet]. Available at:

http://www.culture.gov.uk/what_we_do/Broadcasting/digital_bro adcasting/digital_television.htm [accessed May 2007].

England, E. \& Finney, A. (2002). Managing Multimedia: Project Management for Web and Convergent Media, Book 2: Technical Issues. London: Addison-Wesley.

Enns, N. \& Mackenzie, I. (1998). 'Touchpad-based remote control devices'. Companion Proceedings of the CHI '98, Proceedings on Human Factors in Computing Systems, pp.229230. New York: ACM.

Eronen, L. \& Vuorimaa, P. (2000). 'User Interfaces for Digital Television: a Navigator Case Study'. Proceedings of the working conference on Advanced visual interfaces - AVI 2000. Palermo, Italy, 23-26 May 2000, pp.276-279. New York: ACM Press. 
International Telecommunication Union (2006). Geneva 2006

Agreement [internet]. Available at:

http://www.itu.int/newsroom/press_releases/2006/11.html

[accessed May 2007].

Isobe, T., Fujiwara, M., Kaneta, H., Morita, T. \& Uratani, N.

(2005). 'Development of a TV reception navigation system

personalized with viewing habits', IEEE Transactions on

Consumer Electronics, 51 (2), pp.655-674.

Kamba, T., Elson, S., Harpold, T., Stamper, T. \& Sukaviriya, P. (1996). 'Using small screen space more efficiently'. Conference on Human Factors and Computing Systems, Proceedings of the CHI '96 Conference on Human Factors in Computing Systems, Vancouver, Canada, 13-18 April 1996, pp.383-390. New York: ACM Press.

Nichols, J.W. (2001). 'Using Handhelds as Controls for Everyday Appliances: A Paper Prototype Study'. Conference on Human Factors in Computing Systems CHI 2001 Student Posters, Seattle, WA, 31 March - 5 April 2001, pp.443-444. New York: ACM Press.

Nichols, J., Myers, B., Higgins, M., Hughes, J., Harris, T., Rosenfeld, R. \& Pignol, M. (2002). 'Generating remote control interfaces for complex appliances'. Symposium on User Interface Software and Technology archive, Proceedings of the 15th annual ACM symposium on User interface software and technology, Paris, France, 27-30 October 2002, pp.161-170.

New York, ACM Press.

Park, J., Blythe, M., Monk, A. \& Grayson, D. (2006). 'Sharable digital TV: relating ethnography to design through un-useless product suggestions'. Conference on Human Factors in Computing Systems CHI 2006, Montreal, Canada, 22-27 April 2006, pp.1199-1204. New York, ACM Press.

Robertson, S., Wharton, C., Ashworth, C. \& Franzke, M. (1996). 'Dual device user interface design: PDAs and interactive television'. Conference on Human Factors in Computing Systems CHI 1996, Proceedings of the SIGCHI conference on Human

Factors in computing systems: common ground, Vancouver, Canada, 13-18 April 1996, pp.79-86. New York, ACM Press. Smyth, B. \& Cotter, P. (2000). 'A personalized television listings service'. Communications of the ACM, 43 (8), pp.107111.

Steemens, J. (1998). Changing Channels, the prospect for television in a digital world. Luton, Bedfordshire: John Libby Media.

TV-Anytime (2007). [Internet]. Available at: http://www.tvanytime.org/ [accessed May 2007].

Wobbrock, J., Forlizzi, J., Hudson, S. \& Myers, B. (2002).
'WebThumb: Interaction Techniques for Small-Screen Browsers'. Symposium on User Interface Software and Technology archive, Proceedings of the 15th annual ACM symposium on user interface software and technology, Paris, France, 27-30 October 2002, pp.205-208. New York: ACM Press.

\section{BIOGRAPHY}

Dr Leon Cruickshank is a lecturer and researcher at Brunel University in multimedia and broadcast media. His research interests focus on the interface between technology and usercentred service and interface design. He has led undergraduate degrees in both Multimedia and Broadcast Media and is an external examiner at Salford University.

Dr Emmanuel Tsekleves is a lecturer in multimedia design and technology at Brunel University, and has research interests in multimedia service creation technologies for broadcast and broadband networks.

Roger Whitham is an interaction/user experience designer specializing in user-centric research and development. He is also undertaking a $\mathrm{PhD}$ investigating personal information management.

Professor Annette Hill is the director of research at the University of Westminster's School of Media, Arts and Design. She is an author most recently of Reality TV: Audiences and Popular Factual Television (Routledge, 2004).

Dr Kaoruko Kondo is a research fellow at the University of Westminster; her research interests are in the area of audiences, children and the media, and diasporic media.

\section{ADDRESSES FOR CORRESPONDENCE}

Dr Leon Cruickshank, Senior Lecturer, Imagination at Lancaster, Lancaster Institute for the Contemporary Arts, The Round House, Lancaster University, Lancaster LA1 4YW, UK

Tel: + 44 (0) 1524592736

Email:1.cruickshank@lancaster.ac.uk

Dr Emmanuel Tsekleves, Creative Multimedia Design/Lv 2 Coordinator for MMTD, School of Engineering and Design, Brunel University, Uxbridge UB8 3PH, UK. 
Tel: $+44(0) 1895267379$

Email: Emmanuel.Tsekleves@brunel.ac.uk

Roger Whitham, School of Engineering and Design, Brunel University, Uxbridge UB8 3PH, UK.

Tel: $+44(0) 1895274000$

Email: roger@rogerwhitham.co.uk

Dr Annette Hill, Department of Journalism and Mass

Communication, School of Media, Arts \& Design, Westminster

University, Watford Road, Northwick Park, Harrow, Middlesex

HA 13 TP, UK.

Tel: + 44 (0) 2079115000 extn 4257

Email: hilla@wmin.ac.uk

Dr Kaoruko Kondo, Research Assistant, Communication and Media Research Institute, Westminster University, Watford Road, Northwick Park, Harrow, Middlesex HA1 3TP, UK. Email:kakosv@hotmail.com

\section{ACKNOWLEDGEMENTS}

This research has been carried out through the User-led Interactive Television Services project (UITS), which is funded by WestFocus (http://www.westfocus.org.uk). This is a consortium of universities in West London, UK, and was established to promote transfer networks and mutually beneficial relationships between academia and industry and between industrial partners that otherwise may not have found each other. UITS is part of the Moving Image collaborative group within the creative industries and has a total funding of $£ 100,000$ over two years. It is a collaboration between Brunel University and the University of Westminster. 